\title{
An unexpected image on transesophageal echocardiography: A hyperechogenic ring on the aortic cusp
}

\author{
Transözofageal ekokardiyografide beklenmeyen bir görüntü: \\ Aortik kuspiste hiperekojen bir halka
}

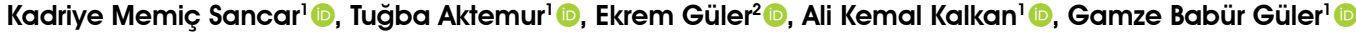 \\ Institution where the research was done: \\ Mehmet Akif Ersoy Thoracic and Cardiovascular Surgery Training and Research Hospital, Istanbul, Turkey
}

Author Affiliations:

'Department of Cardiology, Mehmet Akif Ersoy Thoracic and Cardiovascular Surgery Training and Research Hospital, Istanbul, Turkey ${ }^{2}$ Department of Cardiology, Istanbul Medipol University, Istanbul, Turkey

A 63-year-old male presented to the emergency department with a history of a sudden onset of shortness of breath and chest pain. The patient had prior coronary angiography in an external center due to acute coronary syndrome five years prior to admission. Due to severe left main coronary artery (LMCA) stenosis, a drug-eluting stent from the LMCA to the left anterior descending artery was implanted. Transthoracic echocardiography illustrated a dilated left ventricle (LV) with an estimated ejection fraction of $32 \%$ and moderate-to-severe mitral regurgitation and tachycardia. The patient was directly transferred for coronary angiography. During the procedure, the LMCA could not be cannulated with a Judkins left 4 (JL4) catheter and, therefore, a JL3.5 was used, and only selective images could be taken. Coronary angiography revealed multivessel disease, and coronary artery bypass grafting (CABG) was decided.

Before the operation, transesophageal echocardiography (TEE) was performed to evaluate mitral valve disease. From the aortic valve window, a short-axis view was obtained, and a hyperechoic, fixated, smooth bordered lesion was detected on the left coronary cusp (Figure 1a, Video 1). Visualization of the lesion in the three-dimensional TEE plane from different angles revealed that the lesion was located in sinus of Valsalva. Cusp perforation did not appear in color Doppler imaging (Figure 1b, Video 2) The patient's current and former coronary angiographies were revisited and the LMCA stent was found to protrude into the aorta in both images (Figure 2, Video 3). The Heart Team decided not to remove the stent, since the protruding stent was relatively far from the aortic valve and the part of the stent in LMCA was long and the protruded part of the stent was decided to be measured and re-evaluated using coronary computed tomography prior to operation. Despite having been informed of all the risks, the patient refused CABG and left the hospital after signing the treatment refusal form.

The American College of Cardiology (ACC) and European Society of Cardiology (ESC) guidelines recommend $\mathrm{CABG}$ for patients with Class IB indications in stable LMCA disease that has a favorable coronary anatomy and low surgical mortality.$^{[1]}$ Dislodgement of a stent during or after percutaneous coronary intervention

Received: March 06, 2021 Accepted: July 05, 2021 Published online: October 20, 2021

Correspondence: Kadriye Memiç Sancar, MD. Mehmet Akif Ersoy Göğüs Kalp ve Damar Cerrahisi Eğitim ve Araştırma Hastanesi, Kardiyoloji Kliniği, 34303 Küçükçekmece, İstanbul, Türkiye. Tel: +90 506 - 9139071 e-mail: drkadik@gmail.com 

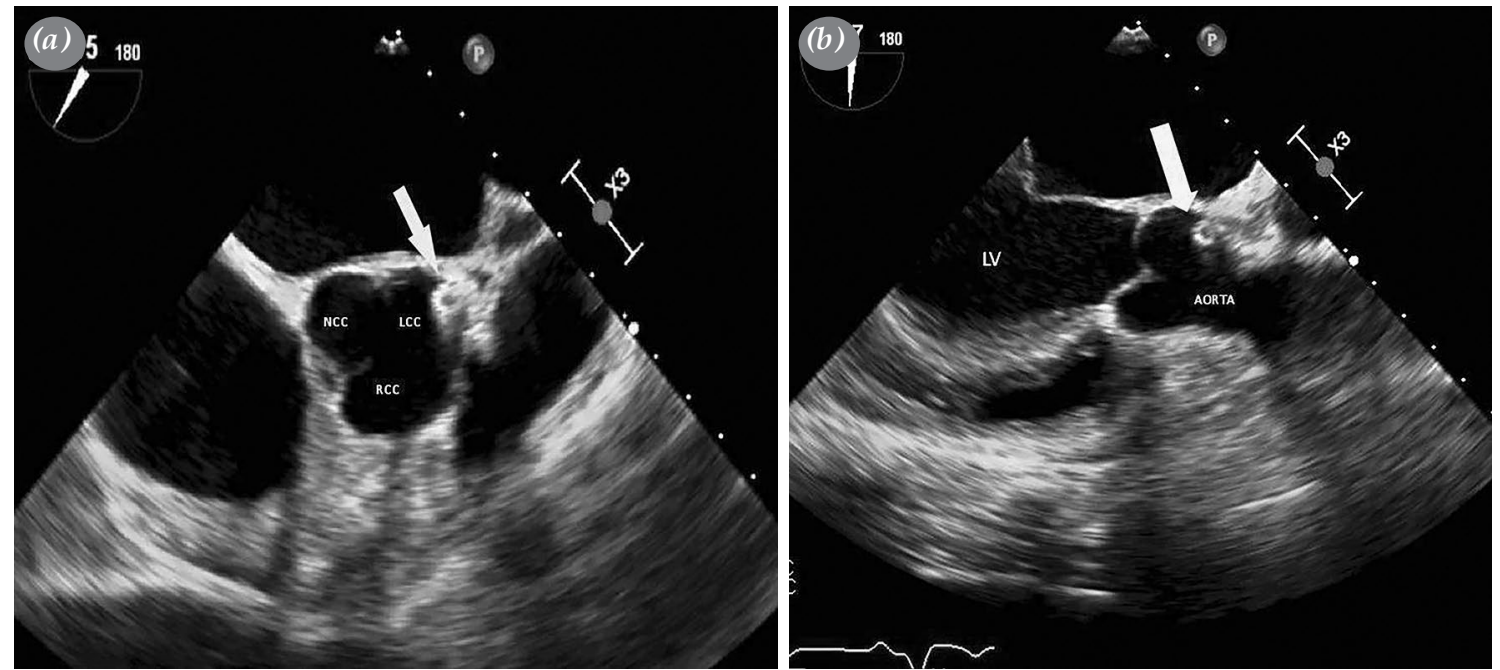

Figure 1. (a) Short axis view of transesophageal echocardiography showing a hyperechoic, fixated, smooth bordered lesion on the left coronary cusp. (b) Transesophageal echocardiographic mid-esophageal aortic valve long-axis view showing fixated, the smooth bordered lesion located in sinus of Valsalva.

LCC: Left coronary cusp; RCC: Right coronary cusp; NCC: Non-coronary cusp; LV: Left ventricle.

is a rare complication with an incidence ranging from 0.32 to $8 \%{ }^{[2]}$ Stent restenosis, stent rupture, thrombosis, and malpositioning are the most common complications during and after percutaneous interventions for the LMCA.$^{[3]}$ The treatment approach for a malpositioned stent is still unclear. A malpositioned LMCA stent can be treated either surgically or conservatively. Gunes et al. ${ }^{[4]}$ presented a case of a successful removal of a malpositioned right coronary artery stent with a guidewire. Also, in two cases presented by Sechi et al., ${ }^{[3]}$ malpositioned and thrombosed LMCA stents were surgically treated. Our case was fortunate enough not to have such complications after stent placement. Although there are different approaches to stent dislodgement treatment, our rare complication case with the silent course demonstrated the importance of detailed echocardiography and multi-modal imaging in the preoperative examination.

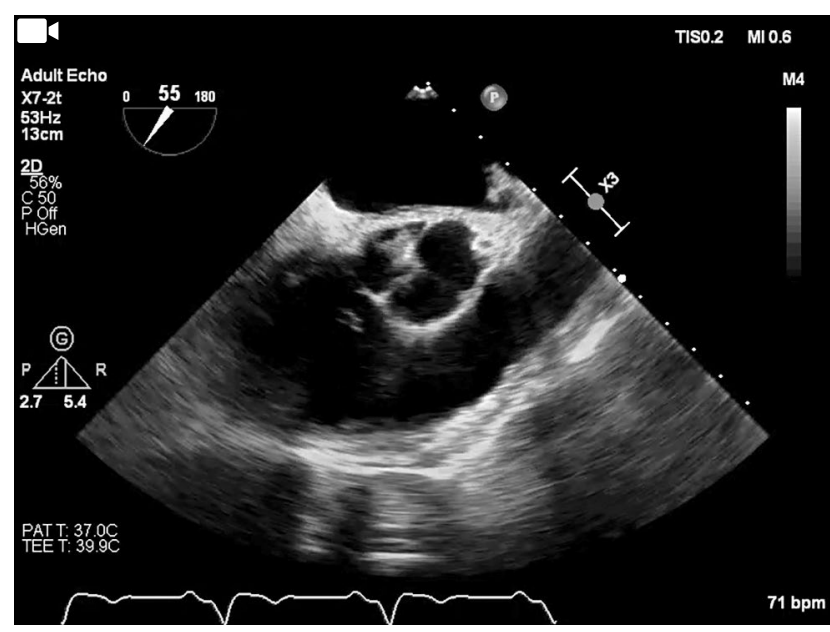

Video 1. Transesophageal echocardiography, short-axis view. A hyperechoic, fixated, smooth bordered lesion on the left coronary cusp is seen.

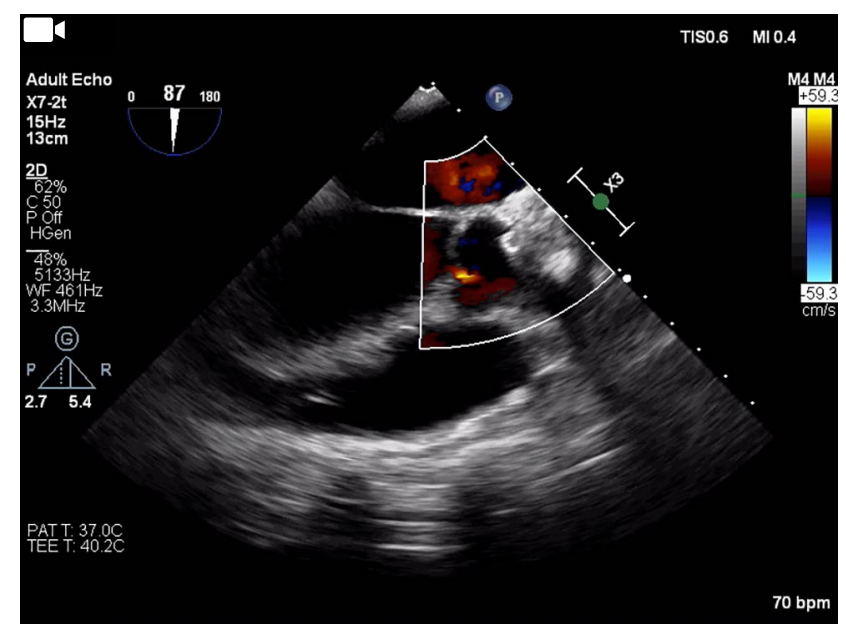

Video 2. Transesophageal echocardiography, mid-esophageal aortic valve long-axis view. Cusp perforation is not shown by color Doppler imaging. 

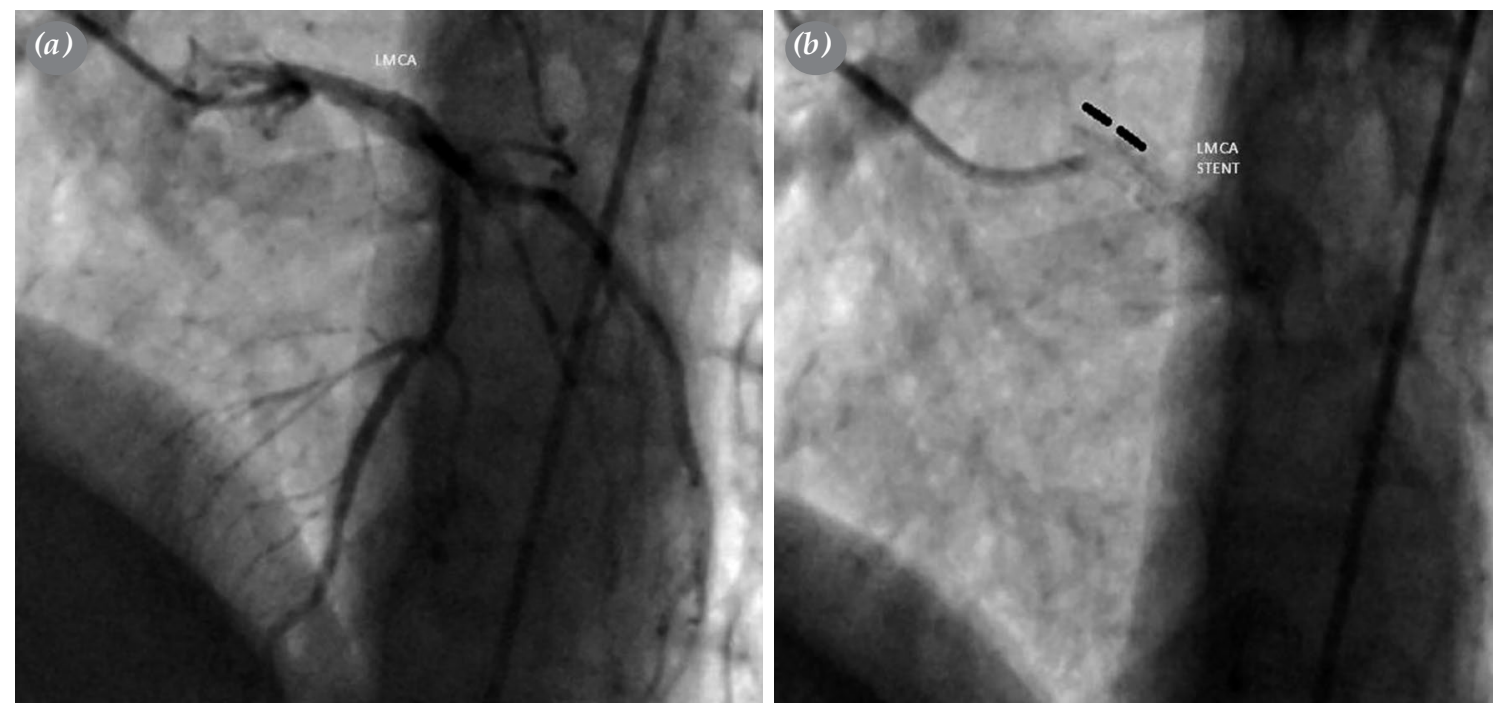

Figure 2. $(\mathbf{a}, \mathbf{b})$ Left coronary angiogram showing the protruded left main stent into the aorta. LMCA: Left main coronary artery.

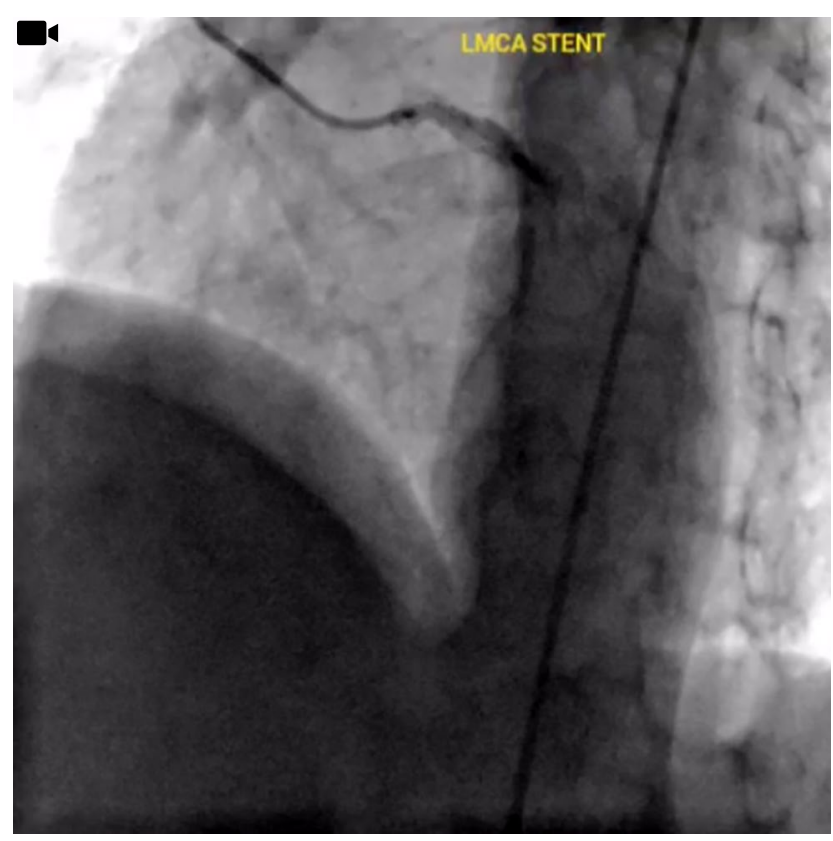

Video 3. Left coronary angiogram view. Protruding part of the LMCA stent became visible after the contrast was washed out. LMCA: Left main coronary artery.

\section{Declaration of conflicting interests}

The authors declared no conflicts of interest with respect to the authorship and/or publication of this article.

\section{Funding}

The authors received no financial support for the research and/or authorship of this article.

\section{REFERENCES}

1. Fajadet J, Capodanno D, Stone GW. Management of left main disease: An update. Eur Heart J 2019;40:1454-66.

2. Jang JH, Woo SI, Yang DH, Park SD, Kim DH, Shin SH. Successful coronary stent retrieval from the ascending aorta using a gooseneck snare kit. Korean J Intern Med 2013;28:481-5.

3. Sechi S, Melina G, Capuano F, Pantanella R, Sinatra R. Left main coronary artery stent misadventure. JACC Case Rep 2020;2:1905-6.

4. Güneş T, Alihanoğlu Yİ, Alur İ, Yıldız BS. Easily removal of a malappositioned coronary stent with a guidewire. Cardiovasc Surg Int 2015;2:47-8. 